\title{
Zur Geographie des Fremdenverkehrs in der Schweiz
}

\section{Einleitung - Entwicklungsphasen des Tourismus}

Der Einfluß des Fremdenverkehrs auf das Landschaftsgefüge hat sich während des letzten Vierteljahrhunderts in progressiver Weise allgemein sichtbar verstärkt. $\mathrm{Er}$ hat Formen angenommen, die gerade auch in den Kreisen des Tourismus Aufsehen erregten und den Mahnruf erwirkten, man möge zur Landschaft als wichtigstem Kapitel des Fremdenverkehrs besser Sorge tragen. Gewiß machte sich der Tourismus, etwa an den Ufern der Voralpenseen oder an klimatisch begünstigten Meeresküsten, schon seit seinen Anfängen im Bilde eines Erdstrichs geltend; Naturschönheit und andere naturgegebene Voraussetzungen trugen neben kulturellen Faktoren wesentlich zu seiner Entfaltung bei. Erst in der jüngern und jüngsten Zeit aber hat er verschiedenenorts in unliebsamer Art gewuchert.

In seiner Geschichte lassen sich verschiedene Phasen unterscheiden. Aus einem vor allem durch Wallfahrten, den Messeverkehr, den Aufenthalt in Heilbädern geprägten Status heraus entwickelte er sich in der Schweiz, zur Epoche der Romantik, zu einem Produktionszweig, der vorerst begeisterte Jünger des Reisens und Wanderns, Freunde der Gebirgswelt hauptsächlich aus begüterten Schichten ergriff und in welchem das Gastgewerbe eine besondere Rolle zu spielen begann. Die bessern Transportmöglichkeiten, die das Dampfschiff und die Eisenbahn schufen, bewirkten zusammen mit der allgemeinen wirtschaftlichen Prosperität einen lebhaften Aufschwung auch des Fremdenverkehrs. Er äußerte sich vor und nach der letzten Jahrhundertwende unter anderem in teilweise vornehm-zurückhaltend sich ins Siedlungsbild fügenden, teilweise aber bereits recht aufdringlichen Hotelbauten. Eine frühe Blüte- und zugleich Pionierzeit des Alpinismus hatte erste, noch bescheidene Bergsteigerzentren erstehen lassen. Ihr folgten nunmehr, dank der Erfindung moderner Mechanismen, welche die Anlage von Schienenwegen in die Bergtäler und bis auf hohe Gipfel hinauf gestatteten, der Einbruch eines immerhin noch überblickbaren Fremdenstromes ins Gebirge und die Bildung von Alpenkurorten und Sommerfrischen in dichterer Streuung.

Einer bedeutsamen Zäsur kam im weitern das Aufkommen des Wintersports gleich. Es brachte in den höher gelegenen Orten den Übergang vom Ein- zum Zweisaisonbetrieb und löste den zu Beginn durchaus maß- vollen Bau weiterer touristischer Einrichtungen aus, wie Eisplätzen,Bobsleigh-Runsodervereinzelten neuen Bergbahnen. Während der u. a. von der Krise der dreißiger Jahre gekennzeichneten Zwischenkriegszeit hielt sich die Entwicklung in engen Grenzen. Erst geraume Zeit nach dem Ende des Zweiten Weltkrieges ging sie in einen Boom über, der alle Voraussagen Lügen strafte. Ursache war die zu schier unaufhörlicher Expansion Anreiz bietende, auch das Reisen und Ferienmachen fördernde Hochkonjunktur. Indem sie dem Automobil als individuellem Verkehrsmittel zu mächtiger Ausbreitung verhalf, schuf sie ganz neue Touristenkategorien. Gleichzeitig begünstigte sie den Bau von Zweitwohnungen in bestehenden oder neu aus dem Boden schießenden Ferienorten. $\mathrm{Zu}$ den traditionellen Unterkunftsstätten gesellten sich die Bauten der sogenannten Parahotellerie, d.h. Ferienchalets, kleinere oder größere Wohn- und Appartementshäuser mit Miet- oder Eigentumswohnungen. Nicht genug damit: Die Attraktionskraft mancher Station schien, nach dem Urteil ihrer Manager, erst durch die Investition zusätzlicher Sportanlagen und -bahnen richtig gewährleistet, und so entstand, neben Schwimmbädern, Kunsteisbahnen usw., ein oft sehr vielfältig verzweigtes Netz von Schwebebahnen und Skilifts, zuweilen bis in die hochalpine Region hinauf. Das Angebot rief dem Zuspruch; zumal an den von den Bevölkerungs-Schwerpunkten nicht allzuweit entfernten Kur- und Sportplätzen entwickelte und entwickelt sich heute zuzeiten ein Massenbetrieb unguter $\mathrm{Di}$ mension.

\section{Fremdenverkehr als Forschungsfach}

\section{Im Rahmen der Wirtschaftswissenschaften}

Die Erforschung des Fremdenverkehrs in seinen wirtschafts- und raumbezogenen Zusammenhängen ist relativ jungen Datums. Während langer Zeit weckte der Tourismus das Interesse der Wissenschafter nur am Rande; auch viele Oekonomen stuften ihn, in einer Aera des Kolonialismus und des aufblühenden Warenhandels, als durchaus sekundär ein. Dies änderte sich mit den durch die beiden Weltkriege im Reise-

Dr. Erich Schwabe, Breichtenstrasse 18, 3074 Muri/BE. 
und Ferienverkehr ausgelösten großen Krisen. Sie öffneten die Augen und verbreiterten das Verständnis für die Bedeutung, die dem Fremdenverkehr als Erwerbszweig wie hinsichtlich der Zahlungsbilanz der einzelnen Staaten mehr und mehr zukam. In der Zwischenkriegszeit setzten denn auch, während kurzer Zeit (1929-1934) vor allem in Deutschland (Berlin), intensivere wissenschaftliche Untersuchungen ein. 1941 entstanden in der Schweiz spezielle, den Hochschulen von Bern und St. Gallen (hier zunächst als Seminar) angeschlossene Forschungsinstitute für Fremdenverkehr; nach 1945 vermehrte sich die Zahl gleich oder ähnlich ausgerichteter Institutionen rasch in ganz Europa und Nordamerika.

Aus praktischen Erwägungen erscheint die Fremdenverkehrsforschung in der Regel den Wirtschaftswissenschaften angegliedert. W. HUNZIKER und K. KRAPF weisen ihr freilich, in ihrem fundamentalen «Grundriß der Allgemeinen Fremdenverkehrslehre» (1942), eine Sonderstellung in der wissenschaftlichen Behandlung zu, denn "sie umfaßt Begriffs- und Erscheinungskomplexe, die außerwirtschaftlicher Natur sind und aus der Wirtschaftstheorie und der wirtschaftlichen Betrachtungsweise heraus allein nicht erklärt und nicht verstanden zu werden vermögen». Die beiden Autoren möchten neben den Wirtschaftswissenschaften auch andere Disziplinen, in gedeihlicher Wechselwirkung, an der Lösung der sich aufdrängenden Fragen beteiligt wissen.

\section{In pluridisziplinärer Sicht}

Aus solchen Überlegungen heraus erfolgte in der Schweiz 1949, auf die Initiative der genannten Theoretiker und zugleich Praktiker des Fremdenverkehrs, der Professoren w. HUNZIKER und K. KRAPF, die Gründung einer Internationalen Vereinigung wissenschaftlicher Fremdenverkehrsexperten (Association internationale d'Experts scientifiques du Tourisme = AIEST). Sie bringt eine größere Zahl qualifizierter Fachleute alljährlich an Tagungen zusammen und verfügt auch über eine eigene, in der Schweiz herausgegebene Zeitschrift, die «Revue de tourisme»/«Zeitschrift für Fremdenverkehn?. Von Anfang an diente sie breitem Informationsaustausch und bildete, sozusagen als Katalysatorin touristischer Forschung, die Plattform für fruchtbare Aussprachen über die vordringlichen Fach- probleme und für ergiebigen Kontakt zwischen den Vertretern verschiedener Wissensbereiche. Diese multidisziplinäre Funktion hat sich mit der Zeit noch verstärkt. Sie trat am jüngsten Kongreß der AIEST, der als 25. Jubiläums-Veranstaltung im September 1975, unter dem Vorsitz von Prof. C. KaSPaR (St. Gallen), in Belgrad stattfand, besonders deutlich zutage. In Partnerschaft hielten Makro- und Mikro-Oekonomen, Geographen und Sozialwissenschafter Rückschau über die Tätigkeit im verflossenen Vierteljahrhundert und suchten daraus für die kommende Arbeit Schlüsse zu ziehen. Der aus den Resultaten und Diskussionen resultierende Bericht umreißt die Bedeutung verschiedener in die Wege geleiteter Studien und Vorkehren; was die Geographie anbelangt, postuliert er für die Zukunft unter anderem die genaue wissenschaftliche Abklärung der geomorphologischen, klimatologischen, biogeographischen und weiterer Umweltfaktoren, die der Ausführung jedes wichtigeren touristischen Erschließungs- oder Ausbauprojektes voranzugehen habe.

\section{Im Rahmen der Geographie}

Vertreter der Geographie - nicht zuletzt aus Frankreich - beteiligten sich schon bei früherer Gelegenheit mehrmals sehr aktiv an der Arbeit der AIEST. Ein bemerkenswertes Grundsatzreferat von P. DEFERT (Paris) bildete 1966 beispielsweise den Ausgangspunkt einer den Standortsfragen des Tourismus gewidmeten Tagung; 1971 galt das Diskussionsthema den Umweltproblemen. 1975 entwickelte sich an den Sitzungen einer besondern Fachgruppe eine lebhafte Aussprache unter - mehrheitlich wiederum französischen - Geographen. Sie baute sich auf einem Vortrag von Mme Y. BARBAZA (Paris) auf, und fand ihre Erfüllung in einem durch B. BARBIER (Aix-Marseille) dem Plenum der Versammlung erstatteten Rapport, in welchem der Beitrag der Landschafts- an die Fremdenverkehrsforschung und -lehre wie die künftigen Aufgaben und Pläne sehr klar umrissen wurden. Dem Basisreferat von Mme BarbaZA und den Ausführungen der weitern Redner und Votanten ließen sich das späte Einsetzen geographischer Analysen des vom Tourismus erfaßten Raumes, der «Fremdenverkehrs-Landschaft», aber auch das jetzt sehr konsequent vorangetriebene Schaffen, mit Hilfe verschiedener Methoden entnehmen. 
Einen ähnlichen Eindruck von der nicht einmal zwei Jahrzehnte alten fremdenverkehrsgeographischen Tätigkeit größern und modernen Stils nahmen auch die Teilnehmer am 40. Deutschen Geographentag mit sich, der im Mai 1975 zu Innsbruck stattfand. Eine von dessen Vortragssitzungen befaßte sich mit der «Geographie des Freizeitverhaltens», in welche K. RUPPERT (München), als einer der Promotoren der Fachrichtung, die Fremdenverkehrsgeographie übergeführt und erweitert sehen möchte. Auch hier vermittelten die verschiedenen Darlegungen die Erkenntnis von jetzt nachhaltig vorangetriebenen Studien. Das gleiche wie für Deutschland und Österreich - und, wie bereits erwähnt wurde, für Frankreich - gilt auch für andere Staaten; als Beleg seien die Akten der vom Frankfurter Geographen J. MATZNETTER geleiteten, der "Geography of Tourism and Recreation» sich widmenden Arbeitsgruppe der International Geographical Union hervorgehoben.

\section{Fremdenverkehrsgeographische Untersuchungen in der Schweiz}

Was wir für drei unserer Nachbarstaaten sagten - der vierte, Italien, macht keine Ausnahme -, läßt sich für unser eigenes Land wiederholen. Auch bei uns begann die landeskundliche Forschung erst längere Zeit nach 1945 sich in konzentrierter Form mit dem Einfluß des Tourismus auf die Landschaft abzugeben; vorangegangen waren Studien mehr regionalgeographischer Art, deren Verfasser das Phänomen "Fremdenverkehn» wohl erwähnten, indessen kaum je auf seine Raumbezüge geschlossen eintraten. - In den späten fünfziger und in den sechziger Jahren kamen die Dinge nacheinander auf mehreren Ebenen ins Rollen. Voran ging das Geographische Institut der Universität Zürich unter der Direktion von Prof. H. BOESCH. Er erkannte als einer der ersten die Bedeutung der vor allem in Nordamerika entwickelten modernen Quantifizierungsmethoden für die Geographie; sein Schüler A. KILCHENMANN wandte sie in seiner Dissertation über die fremdenverkehrs- und wirtschaftsgeographische Struktur der Gemeinden Graubündens an und trug damit ein neues wissenschaftliches System sichtbar nach außen. In der Folge wurde freilich die zur vergleichenden Detailsicht außerordentlich geeignete
Methode bei Arbeiten über Fragen des Tourismus nicht generell zu Hilfe gezogen. Die Untersuchung über die Verflechtung des Fremdenverkehrs Graubündens mit andern landschaftsrelevanten Faktoren, der KILCHENMANN übrigens eine Diplomarbeit über den Fremdenverkehr in Lenzerheide/Valbella hatte vorangehen lassen, regte aber zu weitern Studien in diesem Problembereich an, und es entstand im Zeitraum weniger Jahre am Zürcher Institut eine beachtenswerte Fülle von Zeugnissen aktiver Forschung zur Geographie des Tourismus. Genannt seien hier die Abhandlungen u. a. von P. ALIESCH über den Fremdenverkehr von Chur und über das Stockwerkeigentum im Fremdenverkehr, von R. CALLEGARI über Nendaz, von E. HALDI über Leukerbad, von J. MÜLLER über das Val d'Anniviers, von R. SCHÖNENBER GER über den Oberengadiner Erholungsraum sowie über die Kapazitätsgrenze alpiner Skigebiete, von K. STRUB über Savognin.

Erscheinen die übrigen geographischen Hochschulinstitute, weil andere Fachrichtungen bevorzugend, im Hinblick auf die Fremdenverkehrsforschung eher zurückhaltend - man darf immerhin auf ganz wenige westschweizerische Arbeiten hinweisen -, so haben sich einzelne Fachwissenschafter doch mit Sonderpublikationen hervorgetan; wir denken an die in der Festschrift für H. ANNAHEIM erschienene, am Beispiel von Ronco von W. A. GALLUSSER erstmals in dieser Form beigebrachte Dokumentation der Siedlungsentwicklung und Grundbesitzverhältnisse in der modernen Tessiner Kulturlandschaft. - Aus dem Ausland ist ebenfalls eine Untersuchung über eine schweizerische Fremdenverkehrsgemeinde zu vermelden: die Stuttgarter Dissertation von ARLINDE KRÖNER über Grindelwald, die allerdings ein Schweizer Geograph, Prof. H. GUTERSOHN, mitbetreut hat.

Eine neue Aufgabe ist den Geographen namentlich in den sechziger Jahren seitens der Raumplanung erstanden. Der Fremdenverkehrsgeographie bot sich damit auf einer weitern Ebene Gelegenheit zur Entfaltung. Sie wurde im Rahmen der gesamtschweizerischen landesplanerischen Bemühungen am ORL-Institut der ETH Zürich ergriffen, mit dem das Geographische Institut der ETH, unter den Professoren H. GUTERSOHN und E. WINKLER, in engem Kontakt stand und steht. Die Leistungen gaben sich $u$. a. in den «Landesplanerischen Leitbildern der Schweiz» kund, an denen vonseiten der Geographen, und für die Erfassung des 
Fremdenverkehrs, vor allem auch E. WINKLER mitarbeitete. Zeugnis davon vermittelten deren Schlußbericht und die ihm angefügten Karten, nicht zuletzt jene der Landschaftsstruktur generell, sowie jene der Klimaeignung für Siedlung und Erholung, und der Eignung für Naherholung einerseits, für Ferienerholung und Fremdenverkehr andererseits. - Sodann fand und findet das Schaffen des Geographen auf kantonalem Boden, bei den kantonal- und regionalplanerischen Arbeiten, Gehör und Anerkennung. Vielleicht hier weniger im speziellen Blick auf den Tourismus; und doch sind gerade in diesem Bereich aus dem Kanton Waadt gewichtige Publikationen zu nennen: die umfangreichen und vielfältig dokumentierenden Bände von L. BRIDEL über die Fremdenverkehrsgeographie, über die Skigelände, das Camping und die Freilufterholung.

Eine dritte Plattform für die Darlegung der Probleme des Fremdenverkehrs und der Fremdenverkehrslandschaft ergab sich für die Geographie im großen Werk des «Atlas der Schweiz». Davon soll gesondert die Rede sein.

Schließlich rief das Bedürfnis nach Analyse und Planung von Naherholungsräumen im Umkreis größerer Städte nach neuen wissenschaftlichen Untersuchungen. Diese Sonderform der Fremdenverkehrsforschung steckt noch in den Anfängen. Immerhin hat sie bereits einige interessante Ergebnisse gezeitigt. Die Fremdenverkehrswirtschafter sind an ihnen beteiligt sie haben den Problemen im Herbst 1974 eine eigene, vom Schweiz. Fremdenverkehrsverband veranstaltete Tagung in Wildhaus eingeräumt. Bisher gänzlich getrennt von ihnen arbeiten die Geographen: sie in regionaler Sicht voŕ allem in der Nordwestschweiz. Innerhalb dieses Raumes hat E. BUGMANN die ökologischen und infrastrukturellen Probleme des Erholungsgebietes am Weißenstein bei Solothurn erkundet, während ein Team junger Basler Geographen sich dem Naherholungsbereich des Bruderholzes, im Süden der Rheinstadt, zugewandt hat. Das Geographische Institut der Universität Basel entfaltet in diesem Aufgabenkreis besondere Initiative; es ist vor kurzem von den Behörden von Baselstadt und Baselland mit einer detaillierteren Untersuchung der zwischen dem Jurakamm und der Landesgrenze sich in der Hinsicht stellenden Probleme beauftragt worden. - Das Geographische Institut der Universität Zürich widmet sich in seiner Region dem gleichen Fragenkomplex. Ge- nannt seien die Diplomarbeiten von H.P. SCHWARZ über das Erholungsangebot am und auf dem Zürichsee und von ST. WOTTRENG über die Erholungsgebiete der Stadt Zürich.

\section{Der Tourismus im «Atlas der Schweiz»}

\section{Unterlagen der Karten}

Die beiden der Darstellung des Fremdenverkehrs reservierten Tafeln 64 und 65 des "Atlas der Schweiz», deren Ausarbeitung dem Schreibenden anvertraut war, werteten zum einen statistische Unterlagen aus und suchten zum andern die Struktur von sieben ausgewählten Orten, soweit sie vom Tourismus bestimmt wird, festzuhalten. Wie im Kommentar zur Tafel 64 ausgeführt wird, erschienen kartographische Übersichten des Angebots an touristischen Unterkünften und der Nachfrage nach ihnen als besonders aufschlußreich und wohl auch als vordringlich. Zum mindesten war dies zum Zeitpunkt der Fall, als die Zusammenstellung vorgenommen wurde - zwischen 1964 und 1966. Die seither, bis ins Jahr 1974 hinein sichtbar gewordenen, teilweise außerordentlich aufwendigen und räumlich ausgreifenden Ausbauten und Erweiterungen einzelner Sport- und Ferienstationen haben nicht nur den äußern Aspekt der Ortschaften, sondern auch das für die wissenschaftliche Auswertung geeignete Belegmaterial verändert. So hat die Verankerung des Stockwerkeigentums im Gesetz seit 1965 den Bau mancher großer, oft in übersetztem Chaletstil konstruierter Appartement-Häuser und Wohnblocks gezeitigt. In einer unmittelbar aktuellen Darstellung würde die Ballung des Tourismus in gewissen Orten und Kleinregionen vermutlich noch stärker zutage treten als auf den vorliegenden Karten. Zudem müßte wohl heute auf eine Wiedergabe der räumlichen Präsenz der Fremdenverkehrsplätze im Rahmen ihrer Landschaft vermehrtes Gewicht gelegt werden.

Die statistischen Daten, die für die Haupt- und die beiden Nebenpläne der Tafel 64 zur Verfügung standen, bezogen sich in erster Linie auf das Angebot und die Frequenzen der traditionellen Unterkünfte in Hotels, Pensionen und Kuranstalten. Was die sogenannte Parahotellerie, mit Einschluß der Zweitwohnungen, der Institute, Kinder- und Ferienheime anbelangt, 
hatte das Eidgenössische Statistische Amt, das hinsichtlich der Hotels seit langem sehr zuverlässige Verzeichnisse führte, eben erst begonnen, sie zu sammeln; bis heute liegen indessen keineswegs für die ganze Schweiz, vielmehr nur für die drei Südkantone und den Kanton Bern, regelmäßige Aufzeichnungen vor. Auf kantonaler Basis gab es deren seit längerer Zeit für die Gemeinden Graubündens. In andern Ständen, so in Bern und im Tessin, liefen zur Mitte der sechziger Jahre regelmäßige Erhebungen (Veröffentlichungen im Kanton Bern seit 1965, im Tessin seit 1966). Auf dieses Zahlenmaterial konnte für die Hauptkarte 1:500000 zu einem Teil gegriffen werden. Als weitere Grundlagen für die Erfassung der Parahotellerie dienten einige Belege des Schweizerischen Fremdenverkehrsverbandes sowie eine allerdings lükkenhafte Zusammenstellung des Delegierten für wirtschaftliche Kriegsvorsorge. Im ganzen genügten sie bei weitem nicht. Nur persönliche Erhebungen in zahlreichen Gebieten und Gemeinden vermochten die bestehenden Angaben so zu vervollständigen, daß eine einigermaßen brauchbare Gesamtübersicht geschaffen werden konnte. - Die beiden Nebenkarten in 1:1,1 Million, mit der Darstellung der 1966 in Hotels, Pensionen, Motels und Kuranstalten registrierten Logiernächte sowie der Frequenzen und Ausnützungszahlen in Hotels und vermieteten Ferienwohnungen und -chalets des Berner Oberlandes, vom Herbst 1964 bis Herbst 1965, fußen demgegenüber gänzlich auf den offiziellen statistischen Angaben.

Statistischen Quellen entstammen auch die Eintragungen in den Nebenkarten (Maßstab 1:1,1 Million) der erst mehrere Jahre später herausgegebenen Tafel 65. Einerseits handelte es sich hier darum, die Frequenzen der Bergbahnen eines Fremdenverkehrsplatzes (ohne Skilifte) jeweils zusammengenommen zur Geltung zu bringen. Abgesehen von gewissen Schwierigkeiten, welche sich bei der Datenverwertung von zwei Ferienzentren dienenden Unternehmungen ergaben (Beispiel: Wengernalp- und Jungfraubahn mit Wengen und Grindelwald als Basisorten), verursachte die Tatsache nicht geringe Unsicherheit, daß Jahr für Jahr durch zum Teil wichtige Seilbahn-Verbindungen neue Skisportgebiete sich eröffneten, bei der Darstellung jedoch zeitlich eine Grenze gezogen werden mußte. So kommt auf der Karte etwa den Bahnen am Hoch Ybrig, für welche bei der Auswertung die Frequenzziffern nur einer halben Betriebsperiode zur Verfügung standen, nicht die Bedeutung zu, die sie in Wirklichkeit besitzen. Auch fehlt die frequenzkräftige Sesselbahn im Diemtigtal (Berner Oberland), die wegen des Autobahnanschlusses bei Wimmis ein heute fast zum Naherholungsbereich der Stadt Bern gehörendes Gelände erschließt, aber, weil erst im Winter 1971/72 in Betrieb genommen, nicht berücksichtigt werden konnte. Immerhin vermittelt die Übersicht im gesamten einen Begriff von dem beträchtlichen Verkehrspotential, das an den einzelnen Sportplätzen zur Ausnützung bereit steht, und vom Gebrauch, den die Gäste von ihm machen. Würde man die Skilifte, auf welche die Karte generell nicht Bezug nimmt, sowie die, allerdings nur noch vereinzelt fahrenden Schlittenfunis dazuzählen, so zeichnete sich wahrscheinlich noch verstärkt das Bild einer Konzentration an den Schwerpunkten des Tourismus ab, vielleicht ebensosehr aber auch dasjenige einer Kleinstreuung in abseitigen Tälern, die mit der Anlage eines Skilifts am allgemeinen Boom zu partizipieren hofften.

Die zweite Nebenkarte nimmt sich ein bisher kaum in dieser Art angegangenes Thema vor: Die Besucherzahlen kultureller Stätten wie Museen und Schlösser, sowie von zoologischen und botanischen Gärten, von Höhlen und Schluchtgalerien. Voraussetzung für ihr Erfassen waren Eintrittsbelege; wo solche fehlten und ein Registrieren der Frequenzen sich als unmöglich erwies - etwa bei Kirchen oder Erinnerungsorten (Tellskapelle!) mit freiem Zutritt - mußte von einer Berücksichtigung abgesehen werden. So ergab sich ein etwas einseitiges Bild, indem die Attraktion, welche berühmte Kulturdenkmäler, wie Kathedralen oder Wallfahrtskirchen, auf auswärtige Kunstfreunde oder Pilger ausüben, als nicht meß- und demonstrierbar erschien. Dennoch bietet die Verteilung der Sehenswürdigkeiten, soweit ihnen Rechnung getragen werden konnte, interessante Aspekte genug. Deutlich zeichnet sich eine Massierung der Besucher in den Museen und Tierparks der großen Städte ab. Von den Schlössern erweist sich, nach Chillon und Greyerz als besonders häufig besuchten, eine größere Reihe den Frequenzen nach etwa als gleichwertig. Mit Anziehungspunkten der Natur trumpft besonders das Berner Oberland auf, wobei berühmte Wasserfälle (Gießbach, Staubbach), weil für jedermann frei sichtbar, auf der Karte nicht einmal figurieren - auch der Rheinfall als mächtigster Wassersturz fiel aus dem gleichen Grunde für das Eintragen außer Betracht. 
Abb. 1 Moderner Ausbau der Stadt Lugano in der Cassa-

rate-Talebene und - stark vom Fremdenverkehr, besonders

durch Parahotellerie geprägt - am Hang des Monte Bré.

(Photo svz)

Abb. 2 Sehr umstritten: die junge, willkürliche Überbauung des Geländes von Surlej bei Silvaplana GR, an der Talstation der Corvatsch-Seilbahn. (Photo E. Schwabe)

Abb. 3 Streu-Besiedlung mit Ferienhäusern am Sonnhang von Valbella bei Lenzerheide GR. (Photo svz)
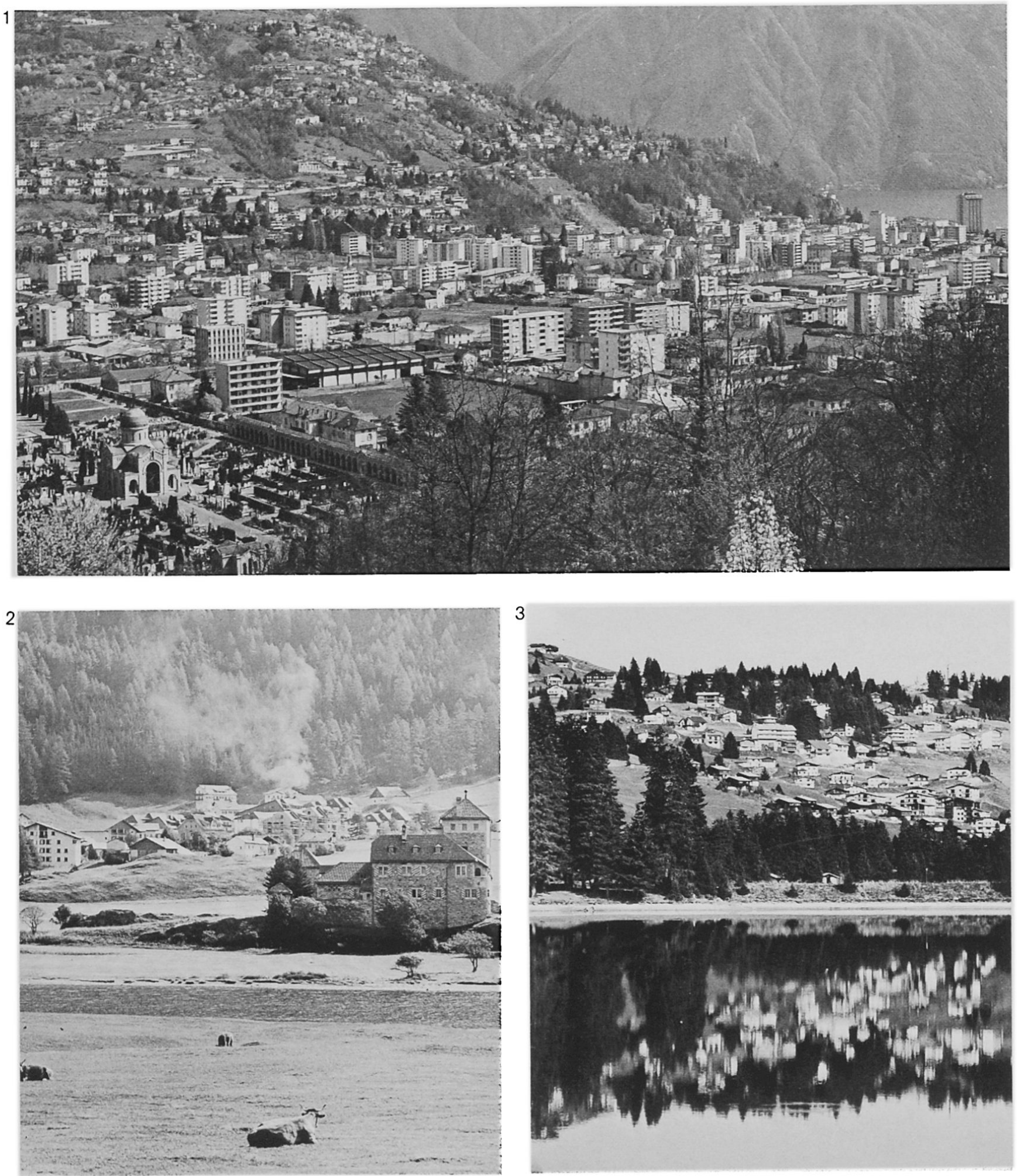
Abb. 4 Adelboden BE dehnt sich mit seinen neuen Ferienhäusern heute von der zentralen Dorfachse aus kompakt hangabwärts. (Photo E. Schwabe)

Abb. 5 Dem Wachstum von Leukerbad VS liegt eine moderne Ortsplanung zugrunde. (Photo svz)

Abb. 6 Die Metropole des Oberengadins, St. Moritz, hat sich namentlich im Bereich ihres Heilbäderquartiers erweitert. (Photo E. Schwabe)
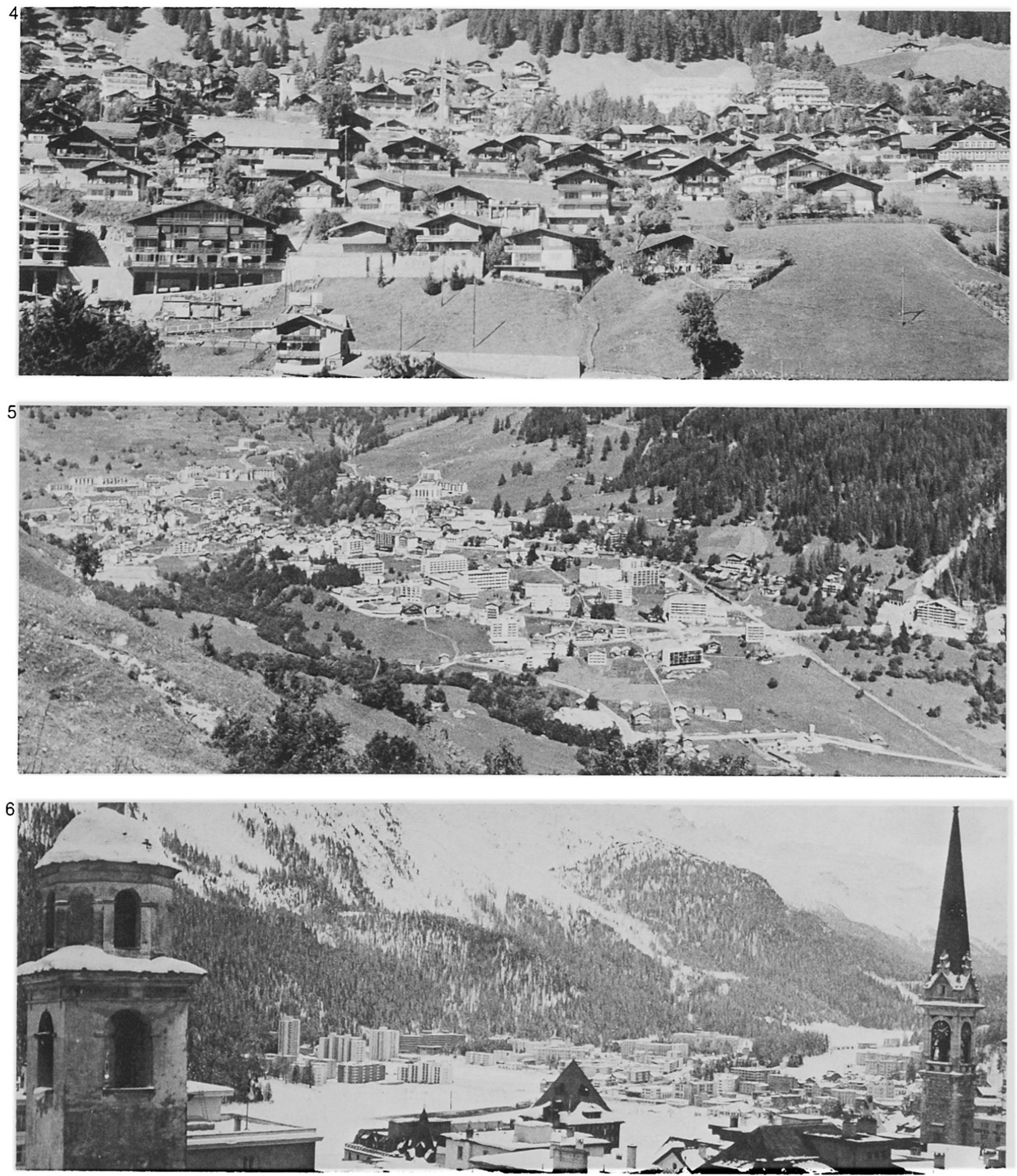
Die Pläne auf den mittleren Seiten von Tafel 65 sind gegenüber den bisher genannten Karten rein geographisch ausgerichtet. Auf der Grundlage vergrößerter Ausschnitte der Landeskarte 1:25000 suchen sie die Siedlungsstruktur von sieben ausgewählten touristischen Zentren und Kurorten wiederzugeben. Als Beispiele dienten: Ascona (Tessin) als Fremdenplatz an einem Seeufer der insubrischen Zone, mit nur einer, aber sehr ausgedehnten Saison; Engelberg (Obwalden) als einstiger Pionierort des Bergsteigens und Wintersports, in alpenrandnaher Lage mit heute sehr starkem Zuzug auch von Wochenendgästen; Leysin (Waadtländeralpen) als ehemaliger rein heilklimatischer Kurort, der sich in einen Sportplatz von ausgesprochen sozialtouristischem Gepräge umgewandelt hat; Verbier (Val de Bagnes, Wallis) als neu erstandenes Ferienzentrum, das von einer großen Menge moderner Chalets, aber auch Appartementblocks, gekennzeichnet wird; Gstaad (Berner Oberland) als Sommer- und Wintersportplatz zum Teil betont mondänen Charakters; Wildhaus (St. Gallen) als beidseits einer stark befahrenen Durchgangsstraße in die Höhe sich ziehender Ferienort eher ländlichen Einschlags; Scuol/SchulsTarasp-Vulpera (Unterengadin, Graubünden) als Heilquellen- und zugleich (Scuol) als Regionalzentrum. Außer Ascona weisen alle Orte Sommer- und Winterbetrieb mit gewissen Besuchsspitzen auf. Bei der Ausarbeitung der Pläne wurde vor allem auf die Kennzeichnung der Gebäude und Anlagen mit touristischer Funktion Wert gelegt. Die gedruckten Karten haben gegenüber den Originalplänen ein leicht reduziertes Bild ergeben. Aus jenen wurde beispielsweise auch die temporäre oder ständige Verwendung von bäuerlichen Heimwesen, oder Teilen von ihnen, für touristische Zwecke (als Ferienwohnungen) ersichtlich; im Falle von Gstaad waren ferner, als konstruktives Detail, moderne Luxus-Chalets mit vorgeblendeten, bemalten und beschnitzten Fassaden abgebrochener Simmentaler- oder saanenländischer Bauernhäuser gesondert gekennzeichnet. Aber auch die vereinfachte Form, wie sie die technische Ausführung verlangte, schafft einen sehr guten Eindruck von dem Gesicht, das der Tourismus in unterschiedlicher Weise, bald mehr mit Hotelbauten, bald mit Ferienhäusern, mit Instituten oder Kinderheimen, auch mit Heilstätten, Badeanlagen usw., neben den Wohnhäusern der Einheimischen und den öffentlichen Gebäuden den einzelnen Siedlungen unverkennbar aufgeprägt hat. -
Der Bearbeiter der Karten möchte nicht unterlassen, hier der Redaktion des «Atlas der Schweiz», vor allem Herrn Prof. E. IMHOF, wie den an der Drucklegung Beteiligten für all ihre Mühewaltung den herzlichsten Dank auszusprechen.

\section{Neue statistische Erhebungen}

Wir haben bereits angetönt, daß eine leichte Verschiebung der Gewichte bei der Darstellung des Fremdenverkehrs durch Geographen künftig wohl am Platze scheint und ihre Berücksichtigung bei einer allfälligen Neuauflage des Atlas, die allerdings noch in der Ferne liegt, sehr zu wünschen wäre. Die landschaftliche Komponente des Tourismus wird in ihrer Bedeutung auch von den Trägern der wirtschaftlich ausgerichteten Fremdenverkehrswissenschaft erkannt und hervorgehoben, wofür u. a. die scharfe Auseinandersetzung des Leiters des Forschungsinstituts für Fremdenverkehr an der Universität Bern, J. KRIPPENDORF, mit den für die wuchernde bauliche Expansion mancher Siedlung Verantwortlichen beredtes Zeugnis ablegt. - Doch sei auch auf die mit der Zeit sich aufdrängende Revision der statistischen Unterlagen hingewiesen. An sie ist ja auch im Hinblick auf andere Karten des Atlas zu denken. Für die verbesserte Deutung des Fremdenverkehrs gilt es vor allen Dingen, exaktere und vollständigere Zahlen aus dem Bereich der Parahotellerie zu beschaffen. Erfreulicherweise liegen heute neue Erhebungen seitens des Instituts für Fremdenverkehr und Verkehrswirtschaft an der Hochschule St. Gallen und seines Leiters C. KASPAR vor. Sie beschränken sich zwar auf vier Kantone - Graubünden, Tessin, Wallis und Waadt -, ziehen in ihnen aber wichtigste Fremdenverkehrsplätze in Untersuchung, von ihnen eine Reihe, die sich durch massiven Verkauf von Grundstücken an Ausländer unrühmlich ausgezeichnet hat.

\section{Ausblick}

A. KILCHENMANN hat mit der zitierten Quantifizierungsmethode einen Weg gewiesen, auf dem sich neue Kenntnisse des Einflusses des Tourismus auf die Struktur der Landschaft und Siedlungen gewinnen, vor allem aber auch treffliche Vergleichsmöglichkeiten erzielen lassen. L. BRIDEL hat bei seinen Untersuchungen 
im Kanton Waadt andere, ihrerseits zu guten Resultaten führende Pfade eingeschlagen. In beiden Fällen war es unerläßlich, ausreichende Daten zur Verfügung zu haben, die als Grundlage dienen konnten. Nicht anders verhielt es sich beim Erarbeiten der Karten des «Atlas der Schweiz».

Die Geographie des Fremdenverkehrs in der Schweiz weiter zu entwickeln, die Verflechtung des Tourismus mit andern landschaftsbildenden Faktoren klärend in den Griff zu bekommen und derart einen Beitrag an das Wissen um Zusammenhänge, und gleichzeitig an das Erfassen der landschaftlichen Werte zu leisten, muß Aufgabe der kommenden Jahre sein. Als vordringlich erscheint dabei, die Unterlagen sicherzustellen, auf denen sich bauen läßt. Vergleichende Übersichten sollten von den verschiedenen Fremdenverkehrsgegenden geschaffen werden. Sie sollten die natürlichen Voraussetzungen - Relief, Boden, Klima, Exposition -, die angestammten Siedlungs- und Bewirtschaftungsverhältnisse, mit den Grenzen der Dauersiedlungen, jenen der Kulturen und des Waldes, mit der Gebäude- und der Landnutzung, wie auch die Grundbesitzverhältnisse festhalten. Parallel dazu sind die Erscheinungen des Tourismus zu registrieren und, in Zusammenarbeit mit den Fremdenverkehrswissenschaftern ökonomischer Richtung, die noch fehlenden statistischen Daten zu beschaffen. Mit der Korrelation all dieser Fakten, in, was uns als sehr wichtig erscheint, steter Fühlungnahme mit Wissenschaftern auch anderer Disziplinen, sollte es möglich sein, der genannten Aufgabe gerecht zu werden.

\section{Recherches touristiques et géographie du tourisme en Suisse}

Pour des raisons pratiques, les recherches touristiques ont été rattachées en général aux sciences économiques. Toutefois, dans leur "Allgemeine Fremdenverkehrslehre», œuvre de base qui a paru en 1942 déjà, les professeurs W. HUNZIKER et K. KRAPF leur ont attribué une position particulière en estimant que d'autres disciplines, parmi lesquelles la géographie, devraient également participer à la solution des problèmes évoqués par le tourisme. L'Association internationale d'experts scientifiques du tourisme (AIEST) créée par la suite, et qui a son centre en Suisse, a toujours fait égard à cette conception. Chaque année, elle réunit des économistes, des géographes, des représentants des sciences sociales et d'autres branches scientifiques en des congrès destinés à discuter à fond, et sur une base interdisciplinaire, des sujets jugés comme les plus actuels.

La géographie du tourisme, en Suisse comme à peu près partout ailleurs, est une branche scientifique particulièrement jeune. Après 1950, et même 1960 seulement, des géographes de notre pays ont commencé de reconnaître la valeur des recherches touristiques. L'initiative était prise notamment par l'Institut de géographie de l'Université de Zurich et son directeur, le professeur H. BOESCH. Par la suite, un véritable essor en thèses et travaux de diplômes, consacrés à des questions que pose le «paysage touristique» s'y est fait sentir. - Indépendemment de ces recherches, le géographe vaudois L. BRIDEL - actuellement président de la commission géographique de la Société helvétique des sciences naturelles - a été chargé par les autorités de son canton d'élaborer un vaste tableau du tourisme vaudois. Ses tomes volumineux forment d'excellents exemples à faire valoir des recherches géographico-touristiques dans le cadre d'un plan d'aménagement. De son côté, l'Institut ORL de l'Ecole polytechnique fédérale à Zurich a toujours eu recours aux géographes, notamment aux professeurs H. GUTERSOHN et E. WINKLER qui se trouvaient parmi ses fondateurs. - En outre, les géographes s'intéressent de plus en plus aussi à des enquêtes sur le tourisme de loisir tel qu'il est pratiqué dans les environs de nos grandes villes. Enfin, un moyen encore de révéler les éléments du tourisme faisant partie de l'ensemble du paysage suisse, s'offrait à eux dans le cadre de l'Atlas de la Suisse. L'étude ci-devant s'occupe en détail des deux planches de l'Atlas consacrées au tourisme suisse et dont l'élaboration était confiée à son auteur. 


\section{Literatur}

AIEST (Association internationale d'experts scientifiques du tourisme). $25^{\mathrm{me}}$ congrès, rapport de synthèse. 1975.

ALIESCH, P.: Der Fremdenverkehr von Chur. Diplomarbeit. Geographisches Institut der Universität Zürich. 1972.

ALIESCH, P.: Das Stockwerkeigentum im Fremdenverkehr. Dissertation. Geographisches Institut der Universität Zürich. 1975.

BARBAZA, Y.: 25 ans de géographie du tourisme. Conférence présentée au $25^{\mathrm{me}}$ congrès de l'AIEST, Belgrade 1975.

BRIDEL, L.: Géographie du tourisme dans le canton de Vaud. Office cantonal vaudois de l'urbanisme. 2 vol. 1970.

BRIDEL, L.: Les champs de ski du canton de Vaud. Office cantonal vaudois de l'urbanisme. 1970.

BRIDEL, L.: Le camping dans le canton de Vaud. Office cantonal vaudois de l'urbanisme. 1970.

BRIDEL, L.: Les loisirs de plein air dans le canton de Vaud. Office cantonal vaudois de l'urbanisme. 1970.

BUGMANN, E.: Oekologische und infrastrukturelle Probleme im Erholungsgebiet Weißenstein. Mitteilungen Naturforschende Gesellschaft Solothurn. 1973.

CALLEGARI, R.: Nendaz. 10 Jahre Fremdenverkehr in einem Walliser Seitental. Diplomarbeit. Geographisches Institut der Universität Zürich. 1973.

DEFERT, P.: La localisation touristique. Publications de l'AIEST, 8. Berne 1967.

FÜGLISTER, H., KÜPFER, D., LÖTSCHER, L.: Das Bruderholz als Naherholungsgebiet. Team-Arbeit des Geographischen Instituts der Universität Basel. Regio basiliensis. 1973.

GALLUSSER, W.: Siedlungsentwicklung und Grundbesitzverhältnisse in der modernen Tessiner Kurlandschaft - eine sozialgeographische Dokumentation über die Gemeinde Ronco s. A. Regio basiliensis. 1968.

Geographie des Freizeitverhaltens. Kurzberichte der Vortragssitzung. 40. Deutscher Geographentag, Innsbruck 1975.

HALDI, E.: Leukerbad. Strukturwandlungen eines Kurorts. Diplomarbeit. Geographisches Institut der Universität Zürich. 1969.
HUNZIKER, W. und KRAPF, K.: Grundriß der Allgemeinen Fremdenverkehrslehre. Polygraphischer Verlag AG Zürich. 1942.

KASPAR, C.: Le bilan des derniers 25 ans de la recherche touristique. Rapport présenté au $25^{\mathrm{e}}$ congrès de l'AIEST, Belgrade 1975.

KASPAR, C.: «Parahotellerie 75», Studie. Institut für Fremdenverkehr und Verkehrswissenschaft an der Hochschule St. Gallen. 1975.

KILCHENMANN, A.: Untersuchungen über den Fremdenverkehr in Lenzerheide/Valbella. Diplomarbeit. Geographisches Institut der Universität Zürich. 1967.

KILCHENMANN, A.: Untersuchungen mit quantitativen Methoden über die fremdenverkehrs- und wirtschaftsgeographische Struktur der Gemeinden im Kanton Graubünden. Dissertation. Juris-Verlag. 1968.

KRIPPENDORF, J.: Die Landschaftsfresser. Tourismus und Erholungslandschaft - Verderben oder Segen? Hallwag AG, Bern. 1975.

KRÖNER, A.: Grindelwald. Die Entwicklung eines Bergbauerndorfes zu einem internationalen Touristenzentrum. Dissertation. Stuttgarter Geographische Studie 74. 1968.

Landesplanerische Leitbilder der Schweiz, Schlußbericht. Schriftenreihe zur Orts-, Regional- und Landesplanung des ORL-Instituts an der ETH Zürich, Nr. $10 \mathrm{~A}$. 1971.

MÜLLER, J.: Untersuchung über die Wandlung der alpinen Wirtschaftsstruktur im Val d'Anniviers. Diplomarbeit. Geographisches Institut der Universität Zürich. 1967.

Naherholungstourismus - Probleme und Lösungen. Berichte zur Arbeitstagung der Dokumentations- und Beratungsstelle des Schweizerischen Fremdenverkehrsverbandes. Wildhaus 1974.

PICHARD, A.: Ollon-Villars - évolution d'une commune de la vallée du Rhône. Etude de géographie humaine. Geographica Helvetiva. 2/1969.

SCHÖNENBERGER, R.: Eine wirtschafts- und insbesondere fremdenverkehrsgeographische Strukturanalyse des Oberengadiner Erholungsraumes im Kanton Graubünden. Diplomarbeit. Geographisches Institut der Universität Zürich. 1970. 
Abb. 7 Anzère-Village, oberhalb Sion, mit seinen Großchalets in der Kernzone, eine reißbrettartig geplante "Retortestation" mit starkem Vorherrschen der Parahotellerie (Photo R. Müllhaupt)

SCHÖNENBERGER, R.: Kapazitätsgrenze alpiner Skigebiete. Eine fremdenverkehrsgeographische Studie. Dissertation. Geographisches Institut der Universität Zürich. 1973.

SCHWABE, E.: Alpine Erholungslandschaft. Geographica Helvetica. 3/1964.

SCHWABE, E.: Atlas der Schweiz. Tafeln Fremdenverkehr I und II. Eidg. Landestopographie Bern. 1968 und 1974.

SCHWABE, E.: Fremdenverkehr und Uferschutz an den großen Voralpenseen. Geographica Helvetica. 3/1969. SCHWARZ, HP.: Das Erholungsangebot am und auf dem Zürichsee. Diplomarbeit. Geographisches Institut der Universität Zürich. 1975.
STRUB, K.: Savognin - eine geographische Strukturanalyse des Fremdenverkehrs. Diplomarbeit. Geographisches Institut der Universität Zürich. 1971.

Studies in the Geography of Tourism. Working Conference of the IGU Working Group, Geography of Tourism and Recreation, Salzburg. Frankfurter Wirtschafts- und Sozialgeographische Schriften, Heft 17, 1974.

WOTTRENG, ST.: Erholungsgebiete der Stadt Zürich 1973. Geographisches Institut der Universität Zürich. 1974.

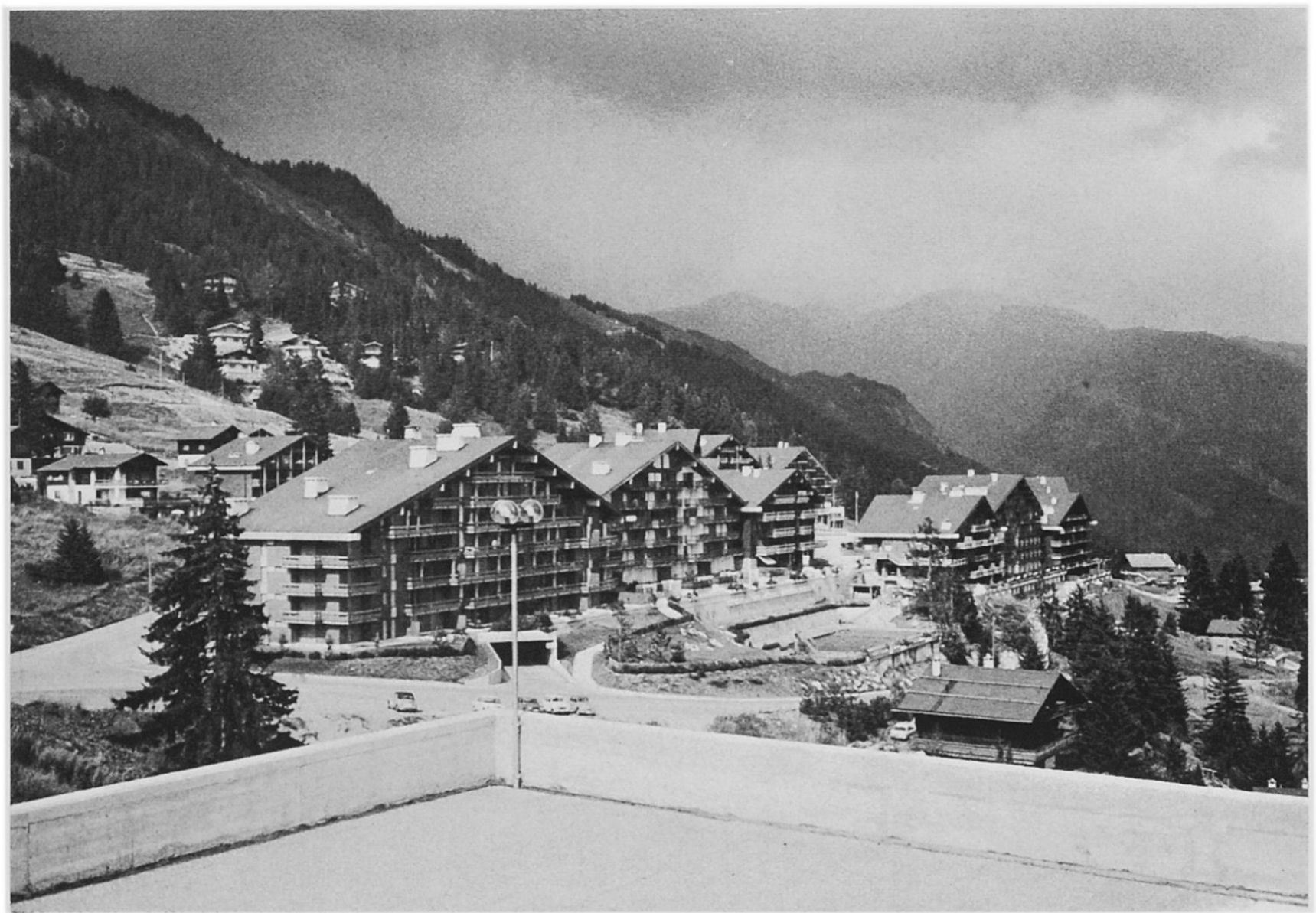

